\title{
ON A CERTAIN CLASS OF NONSTATIONARY SEQUENCES IN HILBERT SPACE
}

\section{BERRABAH BENDOUKHA}

\author{
Received 8 January 2002
}

To my Professor A. A. Yansevitch

\begin{abstract}
We study the functions of correlation $K(n, m)=\langle X(n), X(m)\rangle$ of certain sequences: $X(n)=T^{n} x_{0}, x_{0} \in H$ where $T$ is a contraction in Hilbert space $H$. By using the spectral methods of the nonunitary operators, we give the general form of $K(n, m)$ and its asymptotic behaviour $\lim _{p \rightarrow+\infty} \mathbf{K}(n+p, m+p)$.
\end{abstract}

2000 Mathematics Subject Classification: 47A45, 60G12.

1. Introduction. Let $X(n)(n \in A=I N$ or $Z$ ) be a sequence of elements of a separable Hilbert space $H$. The function of correlation of $X(n)$ is given by formula

$$
K(n, m)=\langle X(n), X(m)\rangle .
$$

If the function of correlation depends only on the difference of arguments, that is, $K(n, m)=K(n-m)$, one calls that $X(n)$ is stationary. Kolmogorov (see [4]) showed that if $X(n)$ is stationary and $A=Z$, then

$$
X(n)=U^{n} x_{0}, \quad x_{0}=X(0),
$$

where $U$ is a unitary operator acting in the subspace $H_{X}$ which is defined as the closed linear envelope of $X=\{X(n) ; n \in Z\}$. This representation as well as the spectral theory of the monoparametric groups of unitary operators allowed to find the general form of the function $K(n, m)$ in the stationary case. More exactly, one has (see [4])

$$
K(n, m)=\int_{-\pi}^{+\pi} e^{i(n-m) \lambda} d F_{X}(\lambda),
$$

where $F_{X}$ is real function, continuous on the left and nondecreasing on $[-\pi ;+\pi]$ such that $F_{X}(-\pi)=0$. This function is called spectral function of $X(n)$.

In this paper, we are interested in some sequences of the form

$$
X(n)=T^{n} x_{0}, \quad x_{0} \in H,
$$


where $T$ is a linear contraction $(\|T\| \leq 1)$ in $H$. Such sequences are called linearly representable and were introduced by Yansevitch $[8,9]$. They represent a natural generalization of the sequences of the form (1.2). But they were especially introduced like the analogue of the processes of the form

$$
Y(t)=e^{i t A} y_{0}
$$

where $A$ is a dissipative $\left(\left(A-A^{*}\right) / i \geq 0\right)$ operator in $H$. The correlation theory of these processes constituted a remarkable field of application for the spectral theory of nonselfadjoint operators [2, 3, 5, 10].

Necessary and sufficient criteria in terms of function of correlation for linear representability (1.4) are established by the following theorem [8].

THEOREM 1.1. A given function $K(n, m)$ is the function of correlation of a certain sequence $X(n)=T^{n} x_{0}$ if and only if there exists a constant $C(0 \prec C \prec$ $+\infty)$ such that

$$
\begin{gathered}
\sum_{n, m=0}^{N} K(n, m) \lambda_{n} \bar{\lambda}_{m} \geq 0, \\
\left|\sum_{n=0}^{N} \sum_{m=0}^{M}(K(n+1, m)-K(n, m)) \lambda_{n} \bar{\mu}_{m}\right|^{2} \\
\leq C \cdot \sum_{n, p=0}^{N} K(n, p) \lambda_{n} \bar{\lambda}_{p} \cdot \sum_{m, q=0}^{M} K(m, q) \mu_{m} \bar{\mu}_{q}
\end{gathered}
$$

for every $\left(\lambda_{n}\right)_{n=0}^{N}$ and $\left(\mu_{m}\right)_{m=0}^{M}$ in the field of complex numbers.

DEFINITION 1.2. Let $X(n)=T^{n} x_{0}$ be a linearly representable sequence. The difference of correlation of $X(n)$ is the function

$$
W(n, m)=K(n, m)-K(n+1, m+1) .
$$

It is clear that in the stationary case, $W(n, m)=0$.

Formula (1.7) implies that, for every natural $p \geq 1$,

$$
K(n, m)=K(n+p, m+p)+\sum_{j=0}^{p-1} W(n+j, m+j),
$$

what gives, for $p \rightarrow+\infty$,

$$
K(n, m)=\lim _{p \rightarrow+\infty} K(n+p, m+p)+\sum_{j=0}^{+\infty} W(n+j, m+j) .
$$


Furthermore, since $\left(I-T^{*} T\right)$ is selfadjoint, then

$$
\left(I-T^{*} T\right)=\sum_{k=1}^{r}\left\langle\cdot ; g_{k}\right\rangle \cdot g_{k}, \quad g_{k} \in\left(I-T^{*} T\right) H, r=\operatorname{dim}\left(I-T^{*} T\right) H .
$$

Hence,

$$
\begin{aligned}
K(n, m) & =\lim _{p \rightarrow+\infty} K(n+p, m+p)+\sum_{j=0}^{+\infty} W(n+j, m+j) \\
& =\lim _{p \rightarrow+\infty} K(n+p, m+p)+\sum_{j=0}^{+\infty}\left\langle\left(I-T^{*} T\right) X(n+j) ; X(m+j)\right\rangle \\
& =\lim _{p \rightarrow+\infty} K(n+p, m+p)+\sum_{j=0}^{+\infty} \sum_{k=1}^{r}\left\langle X(n+j) ; g_{k}\right\rangle \cdot\left\langle g_{k} ; X(m+j)\right\rangle \\
& =\lim _{p \rightarrow+\infty} K(n+p, m+p)+\sum_{j=0}^{+\infty} \sum_{k=1}^{r} \Phi_{k}(n+j) \cdot \overline{\Phi_{k}(m+j)}, \\
\Phi_{k}(n) & =\left\langle X(n) ; g_{k}\right\rangle=\left\langle T^{n} x_{0} ; g_{k}\right\rangle .
\end{aligned}
$$

Consequently, the study of linearly representable sequences can be carried out in two stages.

(a) To find the $\operatorname{limit}_{p \rightarrow+\infty} K(n+p, m+p)$.

(b) To give the explicit expression of the quantity $\Phi_{k}(n)$.

In [8], the case when $\operatorname{dim}\left(I-T^{*} T\right) H=1$ was considered and the spectrum of $T$ is made up only of eigenvalues $\left\{\lambda_{k}\right\}_{k=1}^{+\infty}$ such that $\left|\lambda_{k}\right| \prec 1, k \geq 1$. In this case, one has [8]

$$
\begin{gathered}
\lim _{p \rightarrow+\infty} K(n+p, m+p)=0, \\
K(n, m)=\sum_{j=0}^{+\infty} \Phi(n+j) \cdot \overline{\Phi(m+j)} \\
\Phi(n)=\sum_{k=1}^{+\infty} f_{0 k} \cdot \frac{-1}{2 \pi i} \sqrt{1-\left|\lambda_{k}\right|^{2}} \oint_{\Gamma} \frac{\lambda^{n}}{\lambda-\lambda_{k}} \prod_{j=1}^{k-1} \frac{1-\lambda \cdot \lambda_{j}}{\lambda-\lambda_{j}} \cdot \frac{\left|\lambda_{j}\right|}{\lambda_{j}} \cdot d \lambda, \\
\sum_{k=1}^{+\infty}\left|f_{0 k}\right|^{2} \prec+\infty,
\end{gathered}
$$

where $\Gamma$ is a closed contour containing all the spectrum of $T$.

Let $T$ be a simple contraction (i.e., there is no invariant for $T$ and $T^{*}$ subspace in which, $T$ induces a unitary operator) with spectrum $\sigma(T)$ on the circle unit. It is known [1] that there exists an increasing function $\alpha$ on the interval $[0, l]$ $(l \succ 0)$ such that

$$
\sigma(T)=\left\{e^{-i \alpha(x)}: x \in[0, l]\right\}
$$


DEFINITION 1.3. Say that $X(n)=T^{n} x_{0}$ belongs to the class $D^{(r)}[\alpha]$ if $T$ is a contraction such that (1.13) holds and $\operatorname{dim}\left(I-T^{*} T\right) H \leq r$.

Throughout this paper, we will suppose that $\alpha$ is a continuous function. In this case, we will prove the following results.

THEOREM 1.4. Let $T^{n} x_{0}$ be an element of class $D^{(r)}[\alpha]$. Assume that $T$ is simple. Then,

$$
\begin{gathered}
K(n, m)=\widetilde{\mathbf{K}}_{\infty}(n-m)+F(n-m)+\sum_{j=0}^{+\infty} \sum_{k=1}^{r} \Phi_{k}(n+j) \cdot \overline{\Phi_{k}(m+j),} \\
\Phi_{k}(n)=-\frac{1}{2 \pi i} \oint_{\Gamma} \lambda^{n} \cdot\left\{\frac{\sqrt{2} e^{-x}}{e^{-i \alpha(x)}-\lambda} \int_{0}^{l} \Psi_{0 k}(x) \cdot e^{2 \int_{0}^{x}\left(e^{-i \alpha(t)} / e^{-i \alpha(t)}-\lambda\right) d t} d x\right\} d \lambda,
\end{gathered}
$$

where $\Psi_{0 k} \in L_{[0 ; l]}^{2}, \Gamma$ is any closed contour containing all the spectrum of $T$, $F(n-m)$ is a Hermitian nonnegative function which equals zero in the case when $\operatorname{dim}\left(I-T^{*} T\right) H=1$, and $\widetilde{\mathbf{K}}_{\infty}(n-m)$ is defined by the spectrum of $T$. Moreover, if $T$ has a singular spectrum or the measurement of the intersection of its spectrum with the unit circle is null, then $\widetilde{\mathbf{K}}_{\infty}(n-m)=F(n-m)=0$.

THEOREM 1.5. If a function $K(n, m)$ admits the representation (1.14), then there exists a linearly representable sequence $X(n)=T^{n} x_{0}$ such that $X(n) \in$ $D^{(r)}[\alpha]$ and the function of correlation of $X(n)$ equals $K(n, m)$.

Throughout this paper, $H$ is a separable Hilbert space and $\oplus$ denotes orthogonal sum.

2. On the structure of $\lim _{p \rightarrow+\infty} \mathbf{K}(n+p, m+p)$

Proposition 2.1. If $T$ is a contraction in $H$, then the sequence $A_{n}=T^{* n} T^{n}$ admits a positive strong limit $R=s \cdot \lim _{n \rightarrow+\infty} T^{* n} T^{n}$ which verifies the relation

$$
T^{* n} R T^{m}=R T^{m-n} \quad(n \geq m) .
$$

Moreover, if $T$ is invertible, then

$$
T^{* n} R=R T^{-n} .
$$

Proof. The existence and positivity of $R$ are a consequence of the fact that the sequence $A_{n}$ is a decreasing and bounded sequence of positive operators. Formulas (2.1) and (2.2) are verified easily.

COROLlARY 2.2. If $X(n)=T^{n} x_{0} \in D^{(r)}[\alpha]$, then

$$
\begin{gathered}
\lim _{p \rightarrow+\infty} \mathbf{K}(n+p, m+p)=\mathbf{K}_{\infty}(n-m)=\left\langle R T^{n-m} x_{0}, x_{0}\right\rangle, \\
\lim _{p \rightarrow+\infty} \mathbf{W}(n+p, m+p)=\mathbf{0} .
\end{gathered}
$$


Consider now the sequence

$$
\begin{aligned}
& \hat{\Psi}(x, n)=\widehat{T}^{n} \Psi_{0}(x), \quad \Psi_{0} \in L_{[0 ; l]}^{2}(l \prec \infty), \\
& (\widehat{T} f)(x)=e^{-i \alpha(x)} f(x)-2 e^{-i \alpha(x)+x} \int_{x}^{l} e^{-t} f(t) d t .
\end{aligned}
$$

A direct calculation shows that $\sigma(T)=\left\{e^{-i \alpha(x)}: x \in[0, l]\right\}$ and

$$
\left(I-\hat{T}^{*} \hat{T}\right)=\langle\cdot ; g\rangle \cdot g \quad\left(g(x)=\sqrt{2} e^{-x}\right)
$$

Hence, the sequence $\hat{\Psi}(x, n)$ is an element of the class $D^{(1)}[\alpha]$.

For every $u \in[0, l]$, let

$$
L_{[u ; l]}^{2}=\left\{f \in L_{[0 ; l]}^{2}: f(x)=0 \text { for } x \in[0, l]\right\} .
$$

Let also $P_{u}$ be the orthoprojector of $L_{[0 ; l]}^{2}$ on $L_{[u ; l]}^{2}$.

Proposition 2.3. The sequence $A_{n}(u)=T^{* n} P_{u} T^{n}$ admits a positive strong limit $R_{u}$ which verifies the relation $T^{* n} R_{u} T^{m}=R_{u} T^{m-n}(n \geq m)$. Moreover, if $T$ is invertible, then $T^{* n} R_{u}=R_{u} T^{-n}$.

Pose that

$$
\begin{gathered}
L_{0}(n, u)=\left\langle P_{u}(\hat{\Psi}(x, n)), \hat{\Psi}(x, n)\right\rangle=\int_{u}^{l}|\hat{\Psi}(t, n)|^{2} d t \\
\hat{K}(n, m, u)=\left\langle P_{u}(\hat{\Psi}(x, n)), \hat{\Psi}(x, m)\right\rangle \\
\widehat{W}(n, m, u)=\hat{K}(n, m, u)-\hat{K}(n+1, m+1, u)=y(u, n) \cdot \overline{y(u, m)}, \\
y(u, n)=\sqrt{2} e^{u} \int_{u}^{l} e^{-t} \cdot \hat{\Psi}(t, n) d t .
\end{gathered}
$$

For $n \geq m$,

$$
\begin{aligned}
\widehat{K}(n, m, u) & =\widehat{K}(n-m, 0, u)-\sum_{j=1}^{m} \widehat{W}(n-j, m-j, u) \\
& =\widehat{K}(n-m, 0, u)-\sum_{j=0}^{m-1} \widehat{W}(n-m+j, j, u) .
\end{aligned}
$$

Thus, for $p \geq 1$,

$$
\widehat{K}(n+p, m+p, u)=\hat{K}(n-m, 0, u)-\sum_{j=0}^{m+p-1} \widehat{W}(n-m+j, j, u)
$$


Let $\tau=n-m$ and $\hat{\mathbf{K}}_{\infty}(n-m, u)=\lim _{p \rightarrow+\infty} \hat{\mathbf{K}}(n+p, m+p, u)$. Then,

$$
\hat{\mathbf{K}}_{\infty}(n-m)=\hat{K}(\tau, 0, u)-\sum_{j=0}^{+\infty} y(u, \tau+j) \overline{y(u, j)} .
$$

Let

$$
\mathbf{L}_{p}(\tau, u)=\hat{K}(\tau, 0, u)-\sum_{j=0}^{p-1} y(u, \tau+j) \overline{y(u, j)} \quad(p \geq 1) .
$$

Then,

$$
\begin{gathered}
\hat{\mathbf{K}}_{\infty}(\tau, u)=\lim _{p \rightarrow+\infty} \mathbf{L}_{p}(\tau, u), \\
\mathbf{L}_{p}(0, u)=\hat{K}(0,0, u)-\sum_{j=0}^{p-1} y(u, j) \overline{y(u, j)}=\hat{K}(p, p, u)=L_{0}(p, u), \\
K_{0}(u)=\lim _{p \rightarrow+\infty} \mathbf{L}_{p}(0, u)=\lim _{p \rightarrow+\infty} \mathbf{L}_{0}(p, u)=\left\langle R_{u} \Psi_{0}, \Psi_{0}\right\rangle .
\end{gathered}
$$

THEOREM 2.4. The function $\hat{\mathbf{K}}_{\infty}(\tau, u)$ admits the following representation:

$$
\hat{\mathbf{K}}_{\infty}(\tau, u)=-\int_{u}^{l} e^{i \tau \alpha(x)} d K_{0}(x)
$$

In particular,

$$
\hat{\mathbf{K}}_{\infty}(n-m)=-\int_{0}^{l} e^{i(n-m) \alpha(x)} d K_{0}(x) .
$$

Proof. Remark that $K_{0}$ is a decreasing function. Thus integrals in (2.14) and (2.15) exist. A direct but long calculation makes it possible to affirm that

$$
\begin{aligned}
\frac{d}{d u}\left(\mathbf{L}_{p}(\tau+1, u)\right)= & e^{i \alpha(u)}\left(\frac{d}{d u} \mathbf{L}_{p}(\tau, u)\right)+\sqrt{2} y(u, \tau+p) \overline{y(u, p-1)} \\
& +2 y(u, \tau+p) \overline{\Psi(u, p-1)} .
\end{aligned}
$$

Hence,

$$
\begin{aligned}
\frac{d}{d u}\left(\mathbf{L}_{p}(\tau, u)\right)= & e^{i \tau \alpha(u)}\left(\frac{d}{d u} \mathbf{L}_{0}(p, u)\right) \\
& +\sqrt{2} \overline{y(u, p-1)} \sum_{j=1}^{\tau} e^{i(1-j) \alpha(u)} \cdot y(u, \tau+p-j) \\
& +2 \overline{\Psi(u, p-1)} \sum_{j=1}^{T} e^{i(1-j) \alpha(u)} \cdot y(u, \tau+p-j) .
\end{aligned}
$$


Let

$$
\begin{aligned}
& I_{1}=-\int_{u}^{l} e^{i \tau \alpha(x)}\left(\frac{d}{d x} \mathbf{L}_{0}(p, x)\right) d x \\
& I_{2}=-\sqrt{2} \int_{u}^{l} \overline{y(x, p-1)} \sum_{j=1}^{\tau} e^{i(1-j) \alpha(x)} \cdot y(x, \tau+p-j) d x, \\
& I_{3}=-2 \int_{u}^{l} \overline{\Psi(x, p-1)} \sum_{j=1}^{\tau} e^{i(1-j) \alpha(x)} \cdot y(x, \tau+p-j) d x,
\end{aligned}
$$

then

$$
\mathbf{L}_{p}(\tau, u)=I_{1}+I_{2}+I_{3} .
$$

By using the theorem of Lebesgue about dominated convergence, one can show that $I_{2}=I_{3}=0$. Thus,

$$
\mathbf{L}_{p}(\tau, u)=-\int_{u}^{l} e^{i \tau \alpha(x)} d\left(\mathbf{L}_{0}(p, x)\right) .
$$

Furthermore,

$$
\mathbf{L}_{0}(p, x)=\int_{x}^{l}|\hat{\Psi}(t, p)|^{2} d t
$$

is an absolutely continuous function in $x$. Moreover, since operator $\hat{T}$ is a contraction, then

$$
\mathbf{L}_{0}(p, x)=\int_{x}^{l}|\hat{\Psi}(t, p)|^{2} d t \leq \int_{0}^{l}|\hat{\Psi}(t, p)|^{2} d t \leq\left\|\hat{\Psi}_{0}\right\|^{2} .
$$

That means that the sequence $V_{p}(p \geq 1)$ of total variation of $\mathbf{L}_{0}(p, x)$ on $[0, l]$ is bounded. Moreover, function $e^{i \tau \alpha(x)}$ is continuous. Thus,

$$
\begin{aligned}
\lim _{p \rightarrow+\infty} \mathbf{L}_{p}(\tau, u) & =-\int_{u}^{l} e^{i \tau \alpha(x)} d\left(\lim _{p \rightarrow+\infty} \mathbf{L}_{0}(p, x)\right) \\
& =-\int_{u}^{l} e^{i \tau \alpha(x)} d \mathbf{K}_{0}(x) .
\end{aligned}
$$

It is known [6] that if the $X(n)=T^{n} x_{0} \in D^{(1)}[\alpha]$ and $T$ is simple, then $T=U^{-1} \hat{T} U$ where $U$ is a unitary operator from $H$ into $L_{[0 ; l]}^{2}$. Hence, from Theorem 2.4, the following theorem follows.

THeOrem 2.5. Let $X(n)=T^{n} x_{0} \in D^{(1)}[\alpha]$. Suppose that $T$ is simple. Then, there exists an increasing function $\beta$ on $[0, l]$ such that

$$
\mathbf{K}_{\infty}(n-m)=\lim _{p \rightarrow+\infty} \mathbf{K}(n+p, m+p)=\int_{0}^{l} e^{i(n-m) \alpha(x)} d \beta(x) .
$$


Consider now the space $L_{2}^{r}=L_{[0 ; l]}^{2} \oplus \cdots \oplus L_{[0 ; l]}^{2}(r$ times), with scalar product:

$$
\langle f ; g\rangle_{r}=\sum_{j=1}^{r} \int_{0}^{l} f_{j}(x) \cdot \overline{g_{j}(x)} d x, \quad f=\left(f_{1}, \ldots, f_{r}\right), g=\left(g_{1}, \ldots, g_{r}\right) .
$$

In this space, define the operator $\bar{T}(r)=\hat{T} \oplus \cdots \oplus \hat{T}$ as follows:

$$
(\bar{T}(r))\left(f_{1}, \ldots, f_{r}\right)=\left(\widehat{T} f_{1}, \ldots, \hat{T} f_{r}\right)
$$

Every sequence of the form

$$
\begin{gathered}
\widetilde{\Psi}(x, n)=(\bar{T}(r))^{n}\left(\widetilde{\Psi}_{0}(x)\right)=\left(\widehat{T}^{n}\left(\widetilde{\Psi}_{01}\right), \ldots, \hat{T}^{n}\left(\widetilde{\Psi}_{0 r}\right)\right), \\
\widetilde{\Psi}_{0}=\left(\widetilde{\Psi}_{01}, \ldots, \widetilde{\Psi}_{0 r}\right) \in L_{2}^{r},
\end{gathered}
$$

is an element of class $D^{(r)}[\alpha]$ (see [1]). The following relations hold immediately:

$$
\begin{gathered}
\tilde{\mathbf{K}}_{\infty}(n-m)=\lim _{p \rightarrow+\infty} \tilde{\mathbf{K}}(n+p, m+p)=\sum_{j=1}^{r} \hat{\mathbf{K}}_{\infty}^{(j)}(n-m), \\
\hat{\mathbf{K}}_{\infty}^{(j)}(n-m)=-\int_{0}^{l} e^{i(n-m) \alpha(x)} d K_{0}^{(j)}(x), \\
K_{0}^{(j)}(x)=\left\langle R_{x} \Psi_{0 j}, \Psi_{0 j}\right\rangle, \quad(j=1, \ldots, r) .
\end{gathered}
$$

THEOREM 2.6. Let $X(n)=T^{n} x_{0} \in D^{(r)}[\alpha]$. Suppose that $T$ is simple. Then, there exists $r$ increasing functions $\left\{\beta_{j}\right\}_{j=1}^{r}$ on $[0, l]$ and there exists a Hermitian nonnegative function $F(n-m)$ such that

$$
\mathbf{K}_{\infty}(n-m)=\sum_{j=1}^{r} \int_{0}^{l} e^{i(n-m) \alpha(x)} d \beta_{j}(x)+F(n-m) .
$$

Proof. According to [1], there exists a unitary operator $B$ defined in a Hilbert space $M$ such that operator $T$ is unitarily equivalent to the restriction of operator $\bar{B}(r)=\bar{T}(r) \oplus B$ on a certain invariant subspace $\Theta \subset L_{2}^{r} \oplus M$, that is, $T=U^{-1} \bar{B}(r) U$ where $U$ is a unitary operator from $H$ into $\Theta$. Thus,

$$
\begin{aligned}
K(n+p, m+p) & =\left\langle T^{n+p} x_{0} ; T^{m+p} x_{0}\right\rangle \\
& =\left\langle U^{-1} \bar{B}(r)^{n+p} U\left(x_{0}\right) ; U^{-1} \bar{B}(r)^{m+p} U\left(x_{0}\right)\right\rangle \\
& =\left\langle\bar{B}(r)^{n+p}\left(f_{0}\right) ; \bar{B}(r)^{m+p}\left(f_{0}\right)\right\rangle,
\end{aligned}
$$

where $f_{0}=U\left(x_{0}\right)=\widetilde{\Psi}_{0}+x_{M} \in \Theta\left(\widetilde{\Psi}_{0} \in L_{2}^{r}, x_{M} \in M\right)$. 
Since $\bar{B}(r)^{n}=\bar{T}(r)^{n} \oplus B^{n}$, then

$$
\begin{aligned}
K(n+p, m+p)= & \left\langle\bar{T}(r)^{n+p}\left(\widetilde{\Psi}_{0}\right) ; \bar{T}(r)^{m+p}\left(\tilde{\Psi}_{0}\right)\right\rangle \\
& +\left\langle B^{n+p}\left(x_{M}\right) ; B^{m+p}\left(x_{M}\right)\right\rangle \\
= & \left\langle\bar{T}(r)^{n+p}\left(\widetilde{\Psi}_{0}\right) ; \bar{T}(r)^{m+p}\left(\widetilde{\Psi}_{0}\right)\right\rangle \\
& +\left\langle B^{n-m}\left(x_{M}\right) ; x_{M}\right\rangle .
\end{aligned}
$$

Let $F(n-m)=\left\langle B^{n-m}\left(x_{M}\right) ; x_{M}\right\rangle$. It is clear that function $F(n-m)$ satisfies all conditions of Theorem 2.6. Finally, one has

$$
\begin{aligned}
\mathbf{K}_{\infty}(n-m) & =\lim _{p \rightarrow+\infty} K(n+p, m+p) \\
& =\lim _{p \rightarrow+\infty}\left\langle(\bar{T}(r))^{n+p}\left(\widetilde{\Psi}_{0}\right) ;(\bar{T}(r))^{m+p}\left(\widetilde{\Psi}_{0}\right)\right\rangle+F(n-m) \\
& =\widetilde{\mathbf{K}}_{\infty}(n-m)+F(n-m) .
\end{aligned}
$$

To complete the demonstration, it is enough to notice that

$$
\widetilde{\mathbf{K}}_{\infty}(n-m)=\sum_{j=1}^{r} \hat{\mathbf{K}}_{\infty}^{(j)}(n-m)=\sum_{j=1}^{r} \int_{0}^{l} e^{i(n-m) \alpha(x)} d \beta_{j}(x) .
$$

We now will see two situations where $\mathbf{K}_{\infty}(n-m)=0$.

Proposition 2.7. Let $X(n)=T^{n} x_{0} \in D^{(r)}[\alpha]$. If $T$ is simple and the measurement of the intersection of its spectrum with the circle unit is null, then $\mathbf{K}_{\infty}(n-m)=0$.

Proof. One has

$$
\begin{aligned}
|K(n+p, m+p)|^{2} & =\left|\left\langle T^{n+p} x_{0} ; T^{m+p} x_{0}\right\rangle\right|^{2} \\
& \leq\left\|T^{n+p} x_{0}\right\|^{2} \cdot\left\|T^{m+p} x_{0}\right\|^{2} .
\end{aligned}
$$

Under these assumptions, one has according to [7]

$$
\lim _{p \rightarrow+\infty}\left\|T^{n+p} x_{0}\right\|^{2}=\lim _{p \rightarrow+\infty}\left\|T^{m+p} x_{0}\right\|^{2}=0
$$

THEOREM 2.8. Let $T^{n} x_{0}$ be an element of class $D^{(r)}[\alpha]$ and let $\sigma(t)=$ mes $\cdot\{x \in[0, l]: \alpha(x) \prec t\}, t \in[\alpha(0) ; \alpha(l)]$, be the repartition function of $\alpha$. If $T$ is simple and $\sigma$ singular, then $\mathbf{K}_{\infty}(n-m)=0$.

Proof. Under these assumptions, operator $T$ is unitarily equivalent to operator $\bar{T}(r)$ (see [1]). Thus, $\mathbf{K}_{\infty}(n-m)=\widetilde{\mathbf{K}}_{\infty}(n-m)$. But in this case, the 
characteristical function $\tilde{S}(\lambda)$ of operator $\bar{T}(r)$ satisfies the following relations (see $[1,7])$ :

$$
\begin{aligned}
\operatorname{det} \tilde{S}(\lambda) & =\exp \left\{\int_{0}^{l}\left(e^{i \alpha(t)}+\lambda\right)\left(e^{i \alpha(t)}-\lambda\right)^{-1} d t\right\} \\
& =\exp \left\{-\int_{\alpha(0)}^{\alpha(l)}\left(e^{i t}+\lambda\right)\left(e^{i t}-\lambda\right)^{-1} d \sigma(t)\right\} \\
& =\exp \left\{-\int_{0}^{2 \pi}\left(e^{i t}+\lambda\right)\left(e^{i t}-\lambda\right)^{-1} d \nu(t)\right\},
\end{aligned}
$$

where

$$
v(t)= \begin{cases}\sigma(t), & t \in[0, l], \\ 0, & t \notin[0, l]\end{cases}
$$

is a singular function. Thus det $\tilde{S}(\lambda)$ is an interior function and according to [1], for every $\widetilde{\Psi}=\left(\widetilde{\Psi}_{1}, \ldots, \widetilde{\Psi}_{r}\right) \in L_{2}^{r}, \lim _{p \rightarrow+\infty}\left\|T^{n+p} x_{0}\right\|^{2}=0$. By using the same reasoning that in Proposition 2.7, one shows that $\mathbf{K}_{\infty}(n-m)=\widetilde{\mathbf{K}}_{\infty}(n-m)=0$.

\section{General form of $\mathbf{K}(n, m)$}

THEOREM 3.1. Let $T^{n} x_{0}$ be an element of class $D^{(r)}[\alpha]$. Assume that $T$ is simple. Then,

$$
\begin{gathered}
K(n, m)=\widetilde{\mathbf{K}}_{\infty}(n-m)+F(n-m)+\sum_{j=0}^{+\infty} \sum_{k=1}^{r} \Phi_{k}(n+j) \cdot \overline{\Phi_{k}(m+j)}, \\
\Phi_{k}(n)=\frac{-1}{2 \pi i} \oint_{\Gamma} \lambda^{n} \cdot\left\{\frac{\sqrt{2} e^{-x}}{e^{-i \alpha(x)}-\lambda} \int_{0}^{l} \Psi_{0 k}(x) \cdot e^{2 \int_{0}^{x}\left(e^{-i \alpha(t)} / e^{-i \alpha(t)}-\lambda\right) d t} d x\right\} d \lambda,
\end{gathered}
$$

where $\Psi_{0 k} \in L_{[0 ; l]}^{2}$ and $F(n-m)$ is a Hermitian nonnegative function.

PROoF. Using the same reasoning that in Theorem 2.6, one can affirm that

$$
K(n, m)=\widetilde{K}(n, m)+F(n-m),
$$

where $F(n-m)$ satisfies the conditions of Theorem 3.1. According to (1.11),

$$
\begin{gathered}
\tilde{K}(n, m)=\lim _{p \rightarrow+\infty} K(n+p, m+p)+\sum_{j=0}^{+\infty} \sum_{k=1}^{r} \Phi_{k}(n+j) \cdot \overline{\Phi_{k}(m+j)}, \\
\Phi_{k}(n)=\left\langle(\bar{T}(r))^{n}\left(\widetilde{\Psi}_{0}\right) ; g_{k}\right\rangle, \quad\left(I-(\bar{T}(r))^{*}(\bar{T}(r))\right)=\sum_{k=1}^{r}\left\langle\cdot ; g_{k}\right\rangle \cdot g_{k} .
\end{gathered}
$$


One has (see [1])

$$
g_{k}=h_{k} \cdot e_{k}, \quad h_{k}(x)=\sqrt{2} e^{-x},
$$

where $e_{k}(k=1, \ldots, r)$ is the canonical basic in $C^{r}$. Thus,

$$
\Phi_{k}(n)=\left\langle(\bar{T}(r))^{n}\left(\widetilde{\Psi}_{0}\right) ; g_{k}\right\rangle=\Phi_{k}(n)=\left\langle\widehat{T}^{n}\left(\widetilde{\Psi}_{0 k}\right) ; h_{k}\right\rangle
$$

Since $\widehat{T}$ is bounded, then

$$
\hat{T}^{n}=\frac{-1}{2 \pi i} \oint_{\Gamma} \lambda^{n}(\widehat{T}-\lambda \cdot I)^{-1} d \lambda
$$

where $\Gamma$ is a closed contour containing all the spectrum of $\hat{T}$.

Consequently,

$$
\begin{aligned}
\Phi_{k}(n) & =\frac{-1}{2 \pi i} \oint_{\Gamma}\left\langle\lambda^{n}(\hat{T}-\lambda \cdot I)^{-1}\left(\widetilde{\Psi}_{0 k}\right) ; h_{k}\right\rangle d \lambda \\
& =\frac{-1}{2 \pi i} \oint_{\Gamma} \lambda^{n}\left\langle\tilde{\Psi}_{0 k} ;\left(\hat{T}^{*}-\bar{\lambda} \cdot I\right)^{-1}\left(h_{k}\right)\right\rangle .
\end{aligned}
$$

A direct calculation shows that the form $\Phi_{k}(n)$ is as in (3.1).

Theorem 3.1 admits the following reciprocal.

THEOREM 3.2. If a function $K(n, m)$ admits the representation (3.1), then there exists a linearly representable sequence $X(n)=T^{n} x_{0}$ such that $X(n) \in$ $D^{(r)}[\alpha]$ and the function of correlation of $X(n)$ equals $K(n, m)$.

Proof. Since $F(n-m)$ is a Hermitian nonnegative function, there exists (see [4]) a unitary operator $S$ defined in a Hilbert space $M$ such that

$$
F(n-m)=\left\langle S^{n} x_{M}, S^{m} x_{M}\right\rangle, \quad\left(x_{M} \in M\right) .
$$

By the functions $\alpha$ and $\left\{\widetilde{\Psi}_{0 k}\right\}_{k=1}^{r}$ appearing in representation (3.1), construct, in the space $L_{2}^{r}$, the sequence

$$
\widetilde{\Psi}(x, n)=(\bar{T}(r))^{n}\left(\widetilde{\Psi}_{0}(x)\right)=\left(\hat{T}^{n}\left(\widetilde{\Psi}_{01}\right), \ldots, \hat{T}^{n}\left(\widetilde{\Psi}_{0 r}\right)\right),
$$

where operator $T$ is defined in $L_{[0 ; l]}^{2}$ by formula (2.5). Let $H$ denotes the Hilbert space $L_{r}^{2} \oplus M$ with scalar product:

$$
\left\langle g+Y_{M}, g^{\prime}+Y_{M}^{\prime}\right\rangle=\left\langle g, g^{\prime}\right\rangle_{L_{r}^{2}}+\left\langle Y_{M}, Y_{M}^{\prime}\right\rangle_{M}
$$


In this space, define the operator $T=\bar{T}(r) \oplus S$ by $T\left(g+y_{M}\right)=\bar{T}(r)(g)$ $+S\left(y_{M}\right)$. Operator $T$ is a contraction and $\operatorname{dim}\left(I-T^{*} T\right) H=r$. Thus, the sequence $X(n)=T^{n}\left(f+x_{M}\right)=\bar{X}(n)+S^{n} x_{M}$ is an element of class $D^{(r)}[\alpha]$ whose function of correlation equals the given function $K(n, m)$.

\section{REFERENCES}

[1] B. Bendoukha, Modèles universels de certaines classes d'opérateurs dans un espace de Hilbert [Universal models of certain classes of operators on Hilbert space], Maghreb Math. Rev. 5 (1996), no. 1-2, 1-11 (French).

[2] K. Kirchev and V. A. Zolotarev, Nonstationary curves in Hilbert spaces and their correlation functions. II, Integral Equations Operator Theory 19 (1994), no. 4, 447-457.

[3] _ Nonstationary curves in Hilbert spaces and their correlation functions. I, Integral Equations Operator Theory 19 (1994), no. 3, 270-289.

[4] A. N. Kolmogorov, Stationary sequences in Hilbert's space, Bolletin Moskovskogo Gosudarstvenogo Universiteta. Matematika 2 (1941), no. 6, 1-40 (Russian).

[5] M. S. Livshits and A. A. Yansevitch, Operator Colligations in Hilbert Spaces, John Wiley \& Sons, New York, 1979, translated from Russian.

[6] V. T. Poliatsky, On the reduction of quasi-unitary operators to a triangular form, Dokl. Akad. Nauk SSSR (N.S.) 113 (1957), 756-759 (Russian).

[7] B. Sz.-Nagy and C. Foiaş, Analyse Harmonique des Opérateurs de l'Espace de Hilbert, Akadémiai Kiadó, Budapest, 1967 (French).

[8] A. A. Yantsevich, Nonstationary sequences in Hilbert space. I. Correlation theory, Teor. Funktsiĭ Funktsional. Anal. i Prilozhen. (1986), no. 45, 139-141 (Russian).

[9] _ Nonstationary sequences in Hilbert space. II. Spectral representations, Teor. Funktsiĭ Funktsional. Anal. i Prilozhen. (1986), no. 46, 142-144 (Russian).

[10] V. A. Zolotarev and A. A. Yantsevich, Nonstationary curves in Hilbert spaces and nonlinear operator equations, Operator Theory, Subharmonic Functions, Naukova Dumka, Kiev, 1991, pp. 54-60 (Russian).

Berrabah Bendoukha: Department of Mathematics, University of Mostaganem, 27000 Mostaganem, Algeria

E-mail address: ben_berrabah@yahoo.fr 


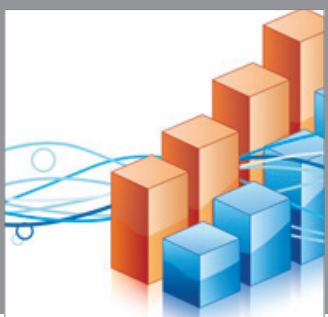

Advances in

Operations Research

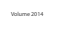

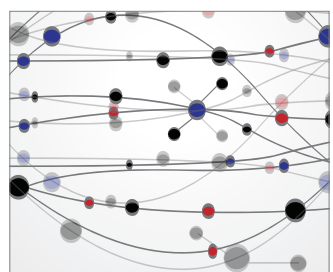

\section{The Scientific} World Journal
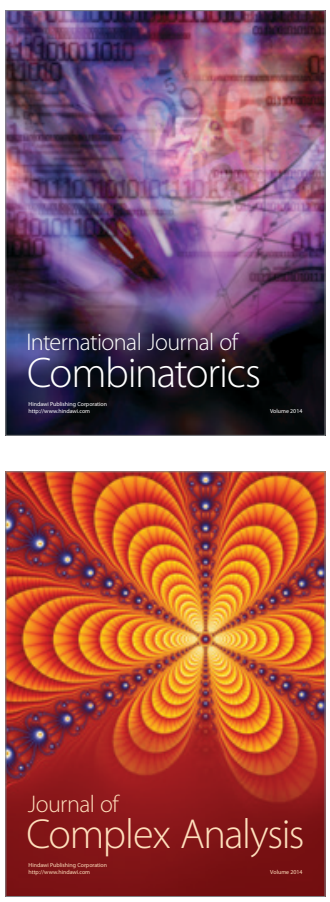

International Journal of

Mathematics and

Mathematical

Sciences
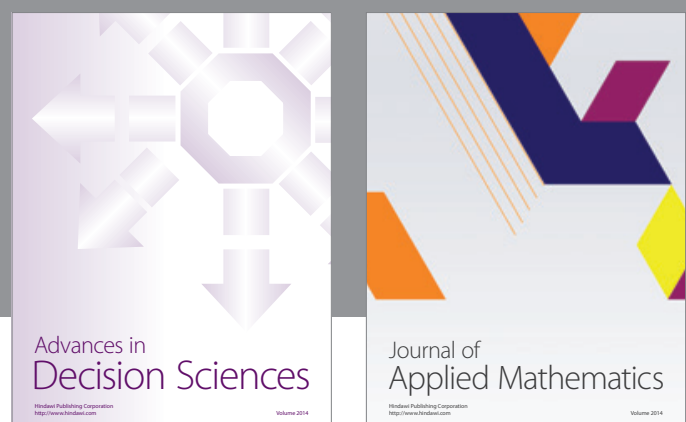

Journal of

Applied Mathematics
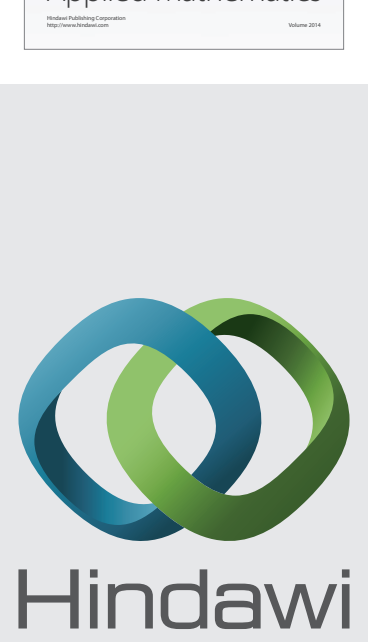

Submit your manuscripts at http://www.hindawi.com
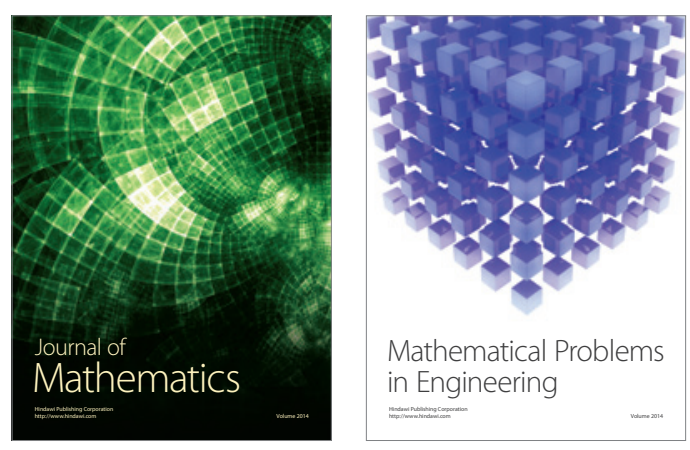

Mathematical Problems in Engineering
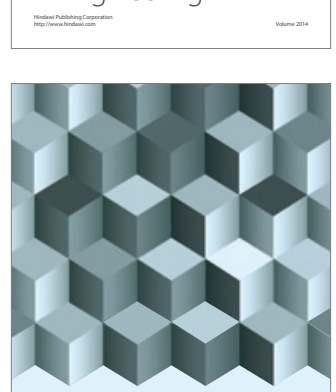

Journal of

Function Spaces
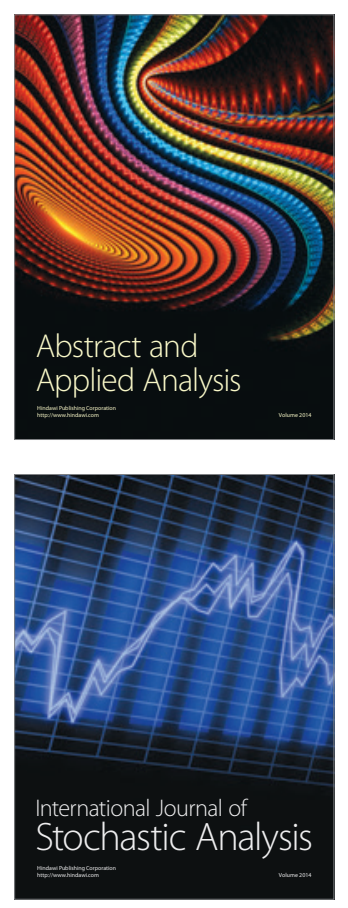

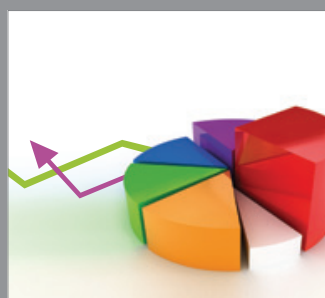

ournal of

Probability and Statistics

Promensencen
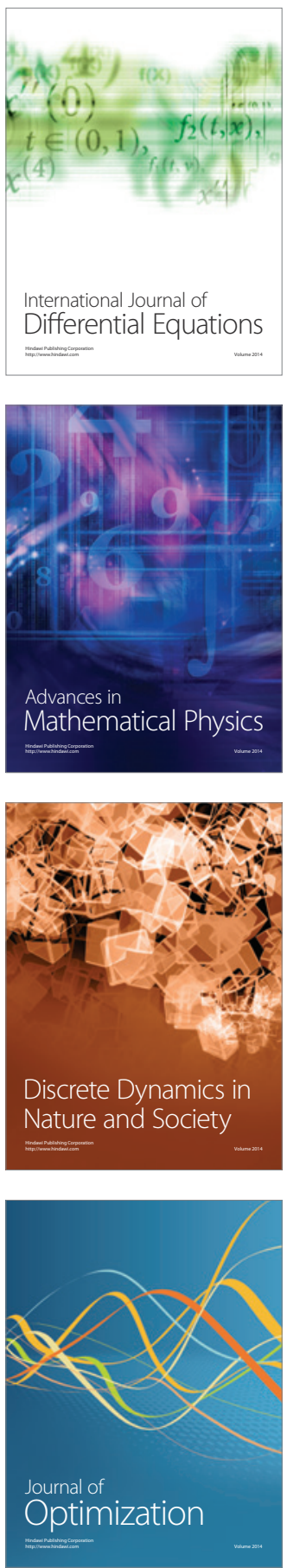